\title{
An identification of the tolerable time-interleaved analog-to- digital converter timing mismatch level in high-speed orthogonal frequency division multiplexing systems
}

\author{
Vo Trung Dung Huynh ${ }^{1,3}$, Linh Mai $^{2,3}$, Hung Ngoc Do ${ }^{1,3}$, Minh Ngoc Truong Nguyen ${ }^{1,3}$, \\ Trung Kien Pham ${ }^{1,3}$ \\ ${ }^{1}$ Department of Telecommunications, School of Electrical Engineering, International University, Ho Chi Minh City, Vietnam \\ ${ }^{2}$ Department of Electronics, School of Electrical Engineering, International University, Ho Chi Minh City, Vietnam \\ ${ }^{3}$ Vietnam National University, Ho Chi Minh City, Vietnam
}

\begin{tabular}{l} 
Article Info \\
\hline Article history: \\
Received Oct 24, 2020 \\
Revised Aug 9, 2021 \\
Accepted Sep 4, 2021 \\
\hline
\end{tabular}

Keywords:

Bit error rate

Orthogonal frequency division

multiplexing

Rayleigh channels

Time-interleaved analog-to-

digital converter

Timing mismatch

\begin{abstract}
High-speed Terahertz communication systems has recently employed orthogonal frequency division multiplexing approach as it provides high spectral efficiency and avoids inter-symbol interference caused by dispersive channels. Such high-speed systems require extremely high-sampling time-interleaved analog-to-digital converters at the receiver. However, timing mismatch of time-interleaved analog-to-digital converters significantly causes system performance degradation. In this paper, to avoid such performance degradation induced by timing mismatch, we theoretically determine maximum tolerable mismatch levels for orthogonal frequency division multiplexing communication systems. To obtain these levels, we first propose an analytical method to derive the bit error rate formula for quadrature and pulse amplitude modulations in Rayleigh fading channels, assuming binary reflected gray code (BRGC) mapping. Further, from the derived bit error rate (BER) expressions, we reveal a threshold of timing mismatch level for which error floors produced by the mismatch will be smaller than a given BER. Simulation results demonstrate that if we preserve mismatch level smaller than $25 \%$ of this obtained threshold, the BER performance degradation is smaller than $0.5 \mathrm{~dB}$ as compared to the case without timing mismatch.
\end{abstract}

This is an open access article under the CC BY-SA license.

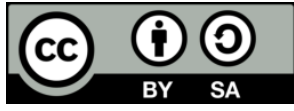

\section{Corresponding Author:}

Vo Trung Dung Huynh

School of Electrical Engineering, International University, Vietnam National University-Ho Chi Minh City Quarter 6, Linh Trung Ward, Thu Duc District, Ho Chi Minh City, Vietnam

Email: hvtdung@hcmiu.edu.vn

\section{INTRODUCTION}

In recent years, orthogonal frequency division multiplexing (OFDM) is a capable multi-carrier modulation technique that has been employed in terahertz $(\mathrm{THz})$ communication systems because it saves transmission bandwidth and provides an ability to transmit high-speed data through dispersive channels [1]-[4]. However, at the receiver, regular analog-to-digital converters (ADCs) cannot operate at such extremely high sampling rates due to the limitation of the current semiconductor technology [5]. To overcome hardware constrains, ADCs with time-interleaved (TI) architecture are usually considered as a practical solution. In general, a time-interleaved (TI-ADC) consists of L parallel sub-ADCs, which simultaneously operate for the same period $T_{s}$. In this fashion, the overall sampling rate of a TI-ADC is L times higher than the sampling rate $\frac{1}{L T_{s}}$ of each sub-ADC. However, due to asymmetric manufacturing in a 
TI-ADC unit, the outcome data can be dramatically degraded by mismatches amongst sub-ADCs. One of the major problems of a TI-ADC is timing mismatch, which is induced by different sampling jitters of subADCs. Over the past decades, timing mismatch's impact and its calibrations were fully researched for singlecarrier communication systems [6]-[17]. In recent past, when OFDM was employed in THz communication systems, this issue has been examined for multi-carrier communication systems, and it was shown that the timing mismatch affects OFDM system performance in a different behavior as compared to single-carrier communication systems [18]-[23]. To the best of our knowledge, the impact of timing mismatch on bit error rate (BER) performance in OFDM systems was investigated by simulation only in most studies, which can be very time consuming. This motivated us to analytically study OFDM-BER performance in the presence of TI-ADC timing mismatch. Further, in practice, the circuits-and-systems designing engineer is interested in determining tolerable timing mismatch levels which induce a negligible system performance degradation. We note that, until now, the identification of tolerable TI-ADC timing mismatch levels has never been investigated for OFDM systems. Hence, in this paper, we will determine tolerable timing mismatch levels of TI-ADCs for OFDM systems in Rayleigh channels. To this end, we derive approximate BER expressions based on the presumption that the interference induced by timing mismatch is Gaussian distributed. Most importantly, the derived BER expressions allow us to reveal a rule-of-thumb for identifying the maximum timing mismatch level, which can be condoned to assure that the performance degradation is smaller than $0.5 \mathrm{~dB}$ as compared to the case without timing mismatch.

The paper is constructed as follows. The BER expressions for square quadrature amplitude modulation (QAM) and pulse amplitude modulation (PAM) are obtained in section 3, assuming binary reflected gray code (BRGC) bit mapping [24]. In section 4, the accuracy of the obtained BER expressions is confirmed by comparing numerical results to Monte Carlo (MC) simulations. In section 5, we reveal a rule-of-thumb for tolerable timing mismatch levels producing an acceptable degradation for fixed timing mismatch and random timing mismatch. Section 6 will conclude the paper.

\section{OFDM SYSTEM MODEL WITH TI-ADC TIMING MISMATCH}

A block diagram of the considered OFDM system employing a TI-ADC at the receiver is shown in Figure 1. In the TI-ADC, the different sub-ADCs will have different timing error values, i.e., $d t_{l}$ denotes the timing error of the $1^{\text {th }}$ sub-ADC. Let the vector $\mathrm{X}$ denote the input of an inverse discrete Fourier transform (IDFT) of size $\mathrm{N}$. Hence, the vector $\mathrm{X}$ consists of $\mathrm{N}$ complexes-values symbols taken from M2-QAM constellation, i.e., $\mathrm{X}=(\mathrm{X} 0, \mathrm{X} 1, \ldots, \mathrm{XN}-1) \mathrm{T}$, with each constellation symbol corresponding to a sequence of $m=\log 2 M$ bits, assuming the BRGC mapping rule [24]. Next, the cyclic prefix (CP) of length NCP is added to the IDFT output to protect the received data against inter-symbol interference (ISI) induced by dispersive channels. The output of the $\mathrm{CP}$ insertion unit $\mathrm{s}_{\mathrm{k}}$ is expressed as (1),

$$
S_{k}=\frac{1}{\sqrt{N}} \sum_{c=0}^{N-1} X_{c} e^{j 2 \pi \frac{c k}{N}}, k=-N_{C P},-N_{C P}+1, \ldots, N-1
$$

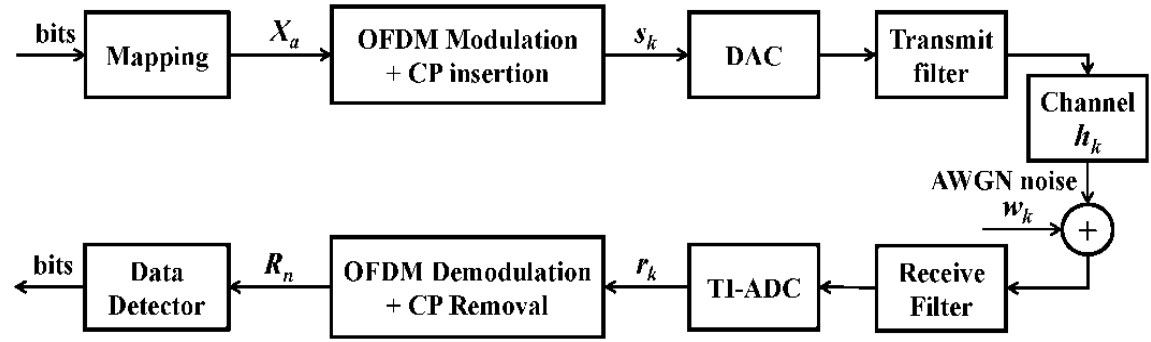

Figure 1. An OFDM system block diagram employs a TI-ADC

Further, a digital-to-analog converter (DAC) at the transmitter will convert the discrete-time signal (1) to a continuous-time signal, which is then shaped by a transmit filter before passing through the channel $h_{k}$ with an additive white Gaussian noise (AWGN) noise wk. At the receiver, the received waveform is passed through a matched receive filter. Then, the $1^{\text {th }}$ sub-ADC samples the resulting signal at sampling time $k L T s+l T s+d t_{l} T s$, where $\mathrm{l}=0,1, \ldots, \mathrm{L}-1$. In our analysis, we assume the TI-ADC to take a sufficiently high resolution in order that the quantization noise is ignored [19]. Moreover, in the most 
practical cases, the timing errors $d t_{l}$ of each sub-ADCs change very slowly in time [19], and hence we can consider their values as constants during an OFDM symbol period. By employing the TI-ADC model affected by timing mismatch in [19], the TI-ADC output is written by (2),

$$
r_{k}=\sqrt{E_{s}} \sum_{m=0}^{N_{C P}-1} h_{m} s_{k-m}\left(d t_{l}\right)+w_{k}, k=-N_{C P},-N_{C P}+1, \ldots, N-1,
$$

where, $E_{s}$ denotes the symbol energy, $l=\bmod (x, y)$ denotes the remainder after division of $x$ by $y, h_{m}$ is the $m^{\text {-th }}$ tap of the sampled impulse response of the channel, $w_{k}$ are independent and identically distributed (i.i.d.) AWGN samples, i.e., $w_{k} \sim \mathrm{N}\left(0, N_{0}\right)$, and $s_{k-m}\left(d t_{l}\right)$ is given by (3)

$$
S_{k-m}\left(d t_{l}\right)=\frac{1}{\sqrt{N}} \sum_{c=0}^{N-1} X_{a} e^{j 2 \pi\left(k-m-d t_{l}\right) \frac{c}{N}}
$$

Substituting (3) into (2), after appropriate re-arrangements, we obtain (4),

$$
r_{k}=\frac{\sqrt{E_{S}}}{\sqrt{N}} \sum_{c=0}^{N-1} X_{c} H_{c} e^{-j 2 \pi \frac{c d t_{l}}{N}} e^{j 2 \pi \frac{c k}{N}}+w_{k}
$$

where, $H_{c}=\sum_{m=0}^{N_{C P}-1} h_{m} e^{-j 2 \pi \frac{m c}{N}}$ denote the frequency channel coefficients. Furthermore, the receiver will remove the $\mathrm{CP}$ and use a discreet Fourier transform (DFT) unit to convert the remaining $N$ samples (from CP removal) to the frequency domain. The DFT output is expressed as (5).

$$
R_{n}=\frac{1}{\sqrt{N}} \sum_{k=0}^{N-1} r_{k} e^{-j 2 \pi \frac{k n}{N}}, n=0,1, \ldots, N-1
$$

To further shorten (5), we consider the concept of a window function $\pi_{k}$, which is defined as $\pi_{k}$ is equal to 1 for $k=0,1, \ldots, N-1$, and is equal to 0 for other cases. Its discrete-time Fourier transform (DTFT) $\Pi(f)$ is defined as $(6)$ :

$$
\Pi(f)=N \sum_{k=-\infty}^{\infty} \operatorname{sinc}(N(f-k)) e^{-j \pi N(f-k)} \approx N \operatorname{sinc}\left(\langle N f\rangle_{N}\right) e^{-j \pi\langle N f\rangle_{N}}
$$

where, $\langle z\rangle_{N}=\bmod (z+N / 2, N)-N / 2$. Further, we substitute (4) into (5), and use the concept of the window function $\pi_{k}$ to change these summations to $k \in[-\infty,+\infty]$, and $q L+l, q \in[-\infty,+\infty], l \in[0,1, \ldots, \mathrm{L}-1]$. Therefore, we have (7):

$$
R_{n}=\frac{\sqrt{E_{S}}}{N} \sum_{c=0}^{N-1} X_{c} H_{c} \sum_{l=0}^{L-1} e^{-j 2 \pi \frac{c d t_{l}}{N}} e^{-j 2 \pi \frac{(n-c) l}{N}} \sum_{q=-\infty}^{+\infty} \pi_{q L+l} e^{-j 2 \pi \frac{(n-c) q L}{N}}+W_{n}
$$

where, $W_{n}=1 / \sqrt{N} \sum_{k=0}^{N-1} W_{k} e^{-j 2 \pi \frac{k n}{N}}$ denotes AWGN samples in the frequency domain with $W_{n} \sim \mathrm{N}\left(0, N_{0}\right)$. Further, we consider the last summation of the first term in (7) as the DTFT of a time-shifted version of $\pi_{k}$. Hence, when we assess this version with $f=\frac{(n-c) L}{N}$, we obtain (8),

$$
e^{-j 2 \pi \frac{(n-c) l}{N}} \sum_{q=-\infty}^{+\infty} \pi_{q L+l} e^{-j 2 \pi \frac{(n-c) q L}{N}}=\frac{1}{L} \sum_{i=0}^{L-1} \Pi\left(\frac{(n-c)}{N}-\frac{i}{L}\right) e^{-j 2 \pi \frac{i l}{L}}
$$

when we substitute (6) and (8) into (7), with proper arrangements, we have (9),

$$
R_{n} \approx \sqrt{E_{s}} T_{n} X_{n} H_{n}+\Psi_{n}, n=0,1, \ldots, N-1
$$

where (9) holds for large $N$, and $T_{n}$ is given by (10),

$$
T_{n}=\sum_{l=0}^{L-1} D T_{i, n} g\left(n-p_{i, n}\right)
$$

with $D T_{z, y}=\frac{1}{L} \sum_{l=0}^{L-1} e^{-j 2 \pi \frac{z d t_{l}}{N}} e^{-j 2 \pi \frac{y l}{N}}, g(z)=\frac{\sin (\pi z)}{\pi z} e^{-j \pi z}$, and $p_{i, n}=\bmod \left(n-i \frac{N}{L}, N\right)$. Further, in (9), $\Psi_{1, n}$ is an interference-plus-noise term, which decomposes as (11):

$$
\Psi_{n}=\Psi_{1, n}+W_{n}
$$


where, $\Psi_{1, n}$ is an inter-carrier interference (ICI) induced by TI-ADC timing mismatch, i.e.,

$$
\Psi_{1, n}=\sqrt{E_{s}} \sum_{i=1}^{L-1} \sum_{c=0, c \neq n}^{N-1} D T_{i, c} X_{c} H_{c} g\left(c-p_{i, n}\right)
$$

Note that with integer ratios $\frac{N}{L}$, we have $T_{n}=D T_{0, n}$ and $\Psi_{1, n}=\sum_{i=0}^{L-1} D T_{i, p_{i, n}} X_{p_{i, n}} H_{p_{i, n}}$. Moreover, assuming a perfect channel estimation, (9) becomes,

$$
\hat{R}_{n}=\sqrt{E_{s}} X_{n}+\frac{\Psi_{n}}{T_{n} H_{n}}
$$

Finally, (13) are used to execute data bit sequence detection.

\section{BER DERIVATION BY USING GAUSSIAN APPROXIMATION}

Assuming that the symbols $\mathrm{X}_{\mathrm{n}}$ can be considered as statistically independent random variables with a uniformly distribution over a normalized-energy constellation, we have: i) $\Psi_{1, n}$ and $W_{n}$ are independent from each other and have zero mean and ii) $\Psi_{1, n}$ is the summation of i.i.d. random variables. In the case of Rayleigh channels (i.e., $H_{n} \sim N\left(0,2 \sigma_{h}^{2}\right)$ ), each variable in the sum (12) is Gaussian distributed. As a result, $\Psi_{1, n}(12)$ has a Gaussian distribution. Hence, the variance $\sigma_{\Psi_{1, n}}^{2}$ of $\Psi_{1, n}$ can be written as (14):

$$
\sigma_{\Psi_{1, n}}^{2}=E_{s} \sum_{i_{1}=1}^{L-1} \sum_{i_{2}=1}^{L-1} \sum_{c=0, c \neq n}^{N-1} D T_{i_{1}, c}\left(D T_{i_{2}, c}\right)^{*} \sigma_{H}^{2} g\left(c-p_{i_{1}, n}\right) g\left(c-p_{i_{2}, n}\right),
$$

where, $(z)^{*}$ implies the conjugate of the complex number $z$. Therefore, the term $\Psi_{n}(11)$ is approximated as a Gaussian random variable, which has zero mean and variance per dimension.

$$
\sigma_{n}^{2}=\sigma_{\Psi_{1, n}}^{2}+\frac{N_{0}}{2}
$$

With the above approximation, for a given symbol Xn, timing mismatch $d t_{l}$ and a given channel $\mathrm{H}$, we can obtain the conditional BER expression in an AWGN channel [21]. Further, in the most practical situations, not all $\mathrm{N}$ sub-carriers are data-modulated, i.e., some sub-carriers nearby the edges are not used to attain an efficient transition guard band [25]. Therefore, assuming only Nd sub-carriers for data transmission $(\mathrm{Nd}<\mathrm{N})$, we can obtain the BER for a given channel $\mathrm{H}$, i.e.,

$$
B E R_{\mid \boldsymbol{H}}=\frac{1}{N_{d} m M} \sum_{n, u, v, y} \alpha_{u, v, y} \operatorname{erfc}\left(\Upsilon_{n, u, v, y}\left(H_{n}\right)\right)
$$

where, the summation runs over $n \in\left\{0,1, \ldots, N_{d}-1\right\} \subset\{0,1, \ldots, N-1\}, u \in\left\{1,2, \ldots, \log _{2} M\right\}, v \in\{0,1, \ldots, M-1\}$ and $y \in\left\{0,1, \ldots, G_{u, v}-1\right\}$. Moreover, in (16), $G_{u, v}$ implies the number of decision boundaries left of the specific constellation symbol, which is given by (17):

$$
G_{u, v}=\left\lfloor(2 v+1) \cdot 2^{-(m-u+2)}+2^{-1}\right\rfloor
$$

where, $\lfloor z\rfloor$ indicates the largest integer smaller than $z$. Furthermore, $\operatorname{erf} c(z)$ in $(16)$ is the complementary error function, and the argument $\Upsilon_{n, u, v, y}\left(H_{n}\right)=\left(S_{v} d-B_{u, y} d\right)\left|T_{n}\right|\left|H_{n}\right| \sqrt{\frac{E_{s}}{2 \sigma_{n}^{2}}}$, with $d$ the half minimum Euclidean distance [26], $S_{v}=2 v+1-M$ and $B_{u, y}=(2 y-1) \quad 2^{m-u+1}-M$. Finally, the pre-factors $\alpha_{u, v, y}=(-1)^{\left\lfloor 2^{u-2-m} \cdot\left(s_{v}-B_{u, y}-1\right)\right\rfloor}$ in (16) take the values +1 or -1 . It can be easily seen that because the BER for the $I$ component or $Q$ component of an $M^{2}$-QAM constellation is similar, (16) also holds for an $M$-PAM modulation. From (16), we need to average the derived BER expression over the statistics of $\left\{\left|H_{n}\right|\right\}$ in order to attain the BER for Rayleigh channels, i.e., $B E R_{\text {Rayleigh }}=\int_{0}^{+\infty} B E R_{\mid \boldsymbol{H}} \times \frac{\left|H_{n}\right|}{\sigma_{H}^{2}} e^{-\frac{\left|H_{n}\right|^{2}}{2 \sigma_{H}^{2}}} d\left|H_{n}\right|$. By using the result in [27], this integral can be further simplified as (18):

$$
B E R=\frac{1}{N_{d} m M} \sum_{n, u, v, y} \alpha_{u, v, y}\left(1-\Theta_{n, u, v, y} \sqrt{\frac{\sigma_{H}^{2} E_{S}}{\sigma_{n}^{2}+\sigma_{H}^{2} E_{S} \Theta_{n, u, v, y}^{2}}}\right)
$$


where, $\Theta_{n, u, v, y}=\left(S_{v} d-B_{u, y} d\right)\left|T_{n}\right|$. It can be obviously seen that (18) grants us to efficiently assess the system performance in the presence of TI-ADC timing mismatch. As a result, this will avoid a demand of very time-consuming $\mathrm{MC}$ simulations to evaluate system performance.

\section{BER EXPRESSION VALIDATION}

In this section, we will confirm the correctness of the obtained BER expressions in a Rayleigh channel by comparing (18) with MC simulations for a variety of constellations and mismatch levels. Further, in our simulations, we assume that all sub-carriers are data-modulated (i.e., $\mathrm{N}=\mathrm{Nd}$ ), and the sampling time $\mathrm{Ts}$ and the symbol energy $\mathrm{E}_{\mathrm{s}}$ are normalized. Moreover, $L^{\prime}$ independent timing errors $d t_{l}^{100 \%}$ are uniformly generated over the interval [-1.1] [19] (see Table 1 with $L=8$ ), which can be represented as the 100\% timing mismatch level of a specific TI-ADC. Futhermore, when $L<L^{\prime}$, only the first L timing errors of the $L^{\prime}$ values in Table 1 are considered in simulations. Furthermore, we define the mismatch level by scaling $d t_{l}^{100 \%}$ in Table 1, i.e., for a mismatch level $z \%$, we have: $d t_{l}^{z \%}=\frac{z}{100} d t_{l}^{100 \%}$. Finally, we consider the model of the channel impulse response in our simulations as (19):

$$
h_{k}=K e^{-\frac{1}{2 \tau}} C_{k}, k=0,1, \ldots, \tau-1
$$

where, $\tau$ denotes the number of channel taps, $C_{k} \sim N(0,1)$, and $K$ is the normalization coefficient such that $\sum_{k=0}^{\tau-1} E\left\{\left|h_{k}\right|^{2}\right\}=1$. In this case, it can be obviously seen that that $H_{n} \sim N(0,1)$.

It can be seen from Figure 2 that a low mismatch level can even induce error floors in the BER performance. Further, Figure 2 shows that these error floors dramatically increase when either the mismatch level or the modulation order increases. Finally, Figures 2(a) and 2(b) reveals that the theoretical and simulated BER curves are in excellent agreement, which confirms the correctness of the obtained BER expressions. We also examined other parameter settings (results are not shown here) and identified the same excellent agreement between theory and simulation. This can be expected as we do not involve any approximation to derive the BER expressions for Rayleigh channels in our analysis.

Table 1. Reference timing errors

\begin{tabular}{cc}
\hline Parameter & Reference values \\
\hline$d t_{l}^{100 \%}$ & {$[-0.92,0.58,0.49,0.79,-0.17,0.7,-0.44,0.76]$} \\
\hline
\end{tabular}

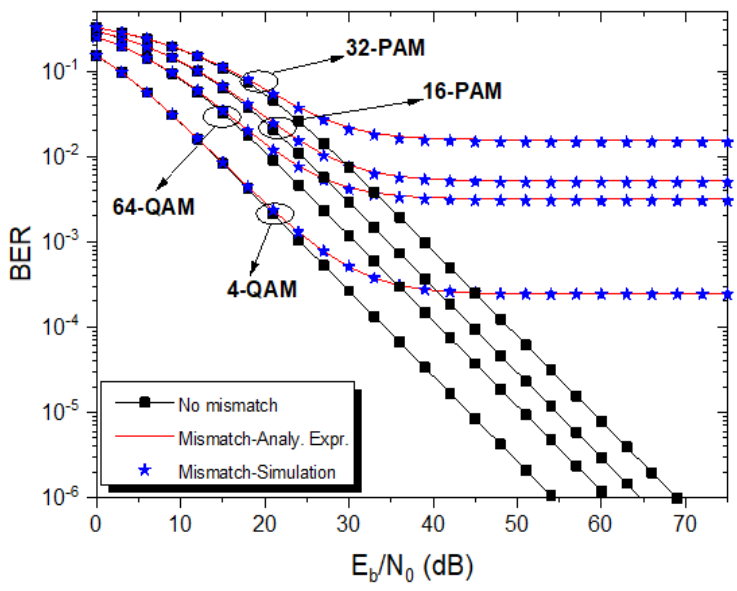

(a)

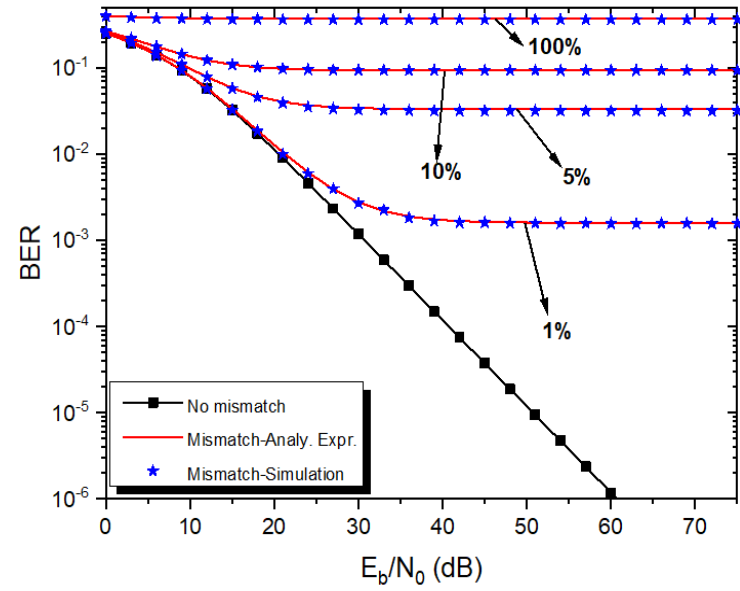

(b)

Figure 2. BER system performance for a Rayleigh channel: (a) $L=8, N=2048$ and $1 \%$ mismatch for different modulation types and (b) 8-PAM, $N=2048$ and $L=7$ with different mismatch levels

\section{AN IDENTIFICATION OF TOLERABLE TIMING MISMATCH LEVELS}

In this section, the tolerable mismatch level inducing a negligible performance degradation will be identified. To this end, we first derive a condition for the maximum timing mismatch level for which error floors induced by timing mismatch will be smaller a given BER value, i.e., $B E R_{t}$. Next, from this derived 
condition, we will reveal a rule-of-thumb to determine tolerable timing mismatch levels at which the system performance degradation is not greater than $0.5 \mathrm{~dB}$ at a given BER value as compared to the mismatch-free case. Section 4 illustrates that the timing mismatch consistently induces the error floor at high SNRs. Further, we interpret the maximum timing mismatch level $\gamma_{R} \%$ for which error floors are lower than $\mathrm{BER}_{\mathrm{t}}$. Moreover, let us define the tolerable timing mismatch level $\tilde{\gamma}_{R} \%$ as the maximum level for which, at BER $\mathrm{t}$, the BER performance degradation is not greater than $0.5 \mathrm{~dB}$ as compared to the case without mismatch. Typically, we have $\tilde{\gamma}_{R} \%<\gamma_{R} \%$. Considering (18), to assure error floors to be lower than $B E R_{t}$ at SNRs, we consider the dominating BER terms only, i.e.,

$$
\frac{B}{N} \sum_{n=0}^{N-1} \max _{u, v, y} \int_{0}^{+\infty} \operatorname{erfc}(\Psi(\gamma \%)) \times \frac{\left|H_{n}\right|}{\sigma_{H}^{2}} e^{-\frac{\left|H_{n}\right|^{2}}{2 \sigma_{H}^{2}}} d\left|H_{n}\right| \leq B E R_{t}
$$

where, $B=\frac{M-1}{M m}, \Psi(\gamma \%)=\left(S_{v} d-B_{u, y} d\right)\left|T_{0, n}^{\gamma \%}\right|\left|H_{n}\right| \sqrt{\frac{E_{S}}{2 \sigma_{\Phi_{1, n}}^{2}(\gamma \%)}}$ and

$$
\begin{aligned}
& \sigma_{\Phi_{1, n}}^{2}(z \%)= \\
& E_{s} \sum_{i_{1}, i_{2} \in\{0,1, \ldots, L-1\} \backslash 0} \sum_{c \in\{0,1, \ldots, N-1\}, c \neq n} D T_{i_{1}, c}^{Z \%}\left(D T_{i_{2}, c}^{Z \%}\right)^{*} \sigma_{H}^{2} f\left(c-p_{i_{1}, n}\right) f\left(c-p_{i_{2}, n}\right)
\end{aligned}
$$

by using the results in [27], (20) can be simplified as (22),

$$
\sum_{n=0}^{N-1} \max _{u, v, y}\left\{1-\sqrt{\frac{\left(S_{v} d-B_{u, y} d\right)^{2}\left|T_{0, n}^{\gamma \%}\right|^{2} \sigma_{H}^{2} E_{S}}{\sigma_{\Phi_{1, n}}^{2}(\gamma \%)+\sigma_{H}^{2} E_{S}\left(S_{v} d-B_{u, y} d\right)^{2}\left|T_{0, n}^{\gamma \%}\right|^{2}}}\right\} \leq \frac{N}{B} B E R_{t}
$$

or equivalently

$$
\sum_{n=0}^{N-1} \min _{u, v, y}\left\{\left(\frac{\sigma_{\Phi_{1, n}}^{2}(\gamma \%)}{\left(S_{v} d-B_{u, y} d\right)^{2}\left|T_{0, n}^{\gamma \%}\right|^{2}}+\sigma_{H}^{2} E_{s}\right)^{-\frac{1}{2}}\right\} \geq J_{R}
$$

where $J_{R}=N\left(\sigma_{H}^{2} E_{S}\right)^{-1 / 2}\left(1-\frac{B E R_{t}}{B}\right)$. From (23), it shows that we minimize the left hand side over $(u, v, y)$ which means minimizing $S_{v} d-B_{u, y} d$ corresponding to $(u, v, y)$. Because $S_{v} d-B_{u, y} d$ denotes a distance between constellation symbols and decision boundaries, we have $\min \left\{S_{v} d-B_{u, y} d\right\}_{u, v, y}=d$. Therefore, (23) becomes (24),

$$
\sum_{n=0}^{N-1}\left\{\left(\frac{\sigma_{\Phi_{1, n}}^{2}\left(\widetilde{\gamma}_{R} \%\right)}{d^{2}\left|\widetilde{\gamma}_{0, n}\right|^{2}}+\sigma_{H}^{2} E_{S}\right)^{-\frac{1}{2}}\right\} \geq J_{R}
$$

Taking into account (24), the threshold $\gamma_{R} \%$, at which error floors produced by the mismatch is lower than $B E R_{t}$, will be next explored, i.e., if we select $d t_{l}^{100 \%}$ in Table 1 as $\gamma \% \times d t_{l}^{100 \%}$ considering $\gamma \% \leq \gamma_{R} \%$ and $B E R_{E_{b} / N_{0} \rightarrow+\infty} \leq B E R_{t}$. To find the threshold $\gamma_{R} \%$, we examine (24) with an equality. From this obtained threshold level $\gamma_{R} \%$, the maximum mismatch level $\tilde{\gamma}_{R} \%$, which induces a performance degradation not greater than $0.5 \mathrm{~dB}$ as compared to the case without mismatch, will be determined. Figure 3(a) demonstrates that in all considered cases, if $\tilde{\gamma}_{R} \%<0.25 \gamma_{R} \%$, there is an acceptable performance degradation $(<0.5 \mathrm{~dB})$ at $B E R_{t}=10^{-9}$. Further, Figure 3(a) also illustrates that if the mismatch level increases to $\gamma_{R} \%$, there is a noticeable performance degradation. We also investigated for other number of sub-ADCs, and other modulation types and orders, and found the similar results. As a result, we can conclude a rule-of-thumb for tolerable timing mismatch levels as (25).

$$
\tilde{\gamma}_{R} \% \leq 0.25 \gamma_{R} \%
$$

Until now, we obtained the results with fix values $d t_{l}$ only. Nevertheless, in the most practical situations, the timing error values $d t_{l}$ are random. Therefore, we will assess the average BER performance with random values $d t_{l}$. This can be attained by averaging the BER system performance over the larger number of different TI-ADCs. In the following results, the timing error values $d t_{l}$ are uniformly chosen over $[-\mathrm{z} / 100, \mathrm{z} / 100], 0 \leq \mathrm{z} \leq 100$, where $\mathrm{z}$ determines the mismatch level in percentage. Further, in each simulation, 
we will choose different sets of $\mathrm{L}$ timing error values $d t l$. Moreover, the results in Figure 3(b) are obtained by employing 1010 TI-ADC realizations, $B E R_{t}=10^{-9}$, and different mismatch levels. It can be seen from Figure 3(b) that the theoretical and simulated BER curves do match well in a Rayleigh channel. Most importantly, Figure 3(b) demonstrates that at $B E R_{t}$, if the mismatch level is equal to the tolerable threshold, i.e., $0.25 \gamma_{R} \%$, where $\gamma_{R} \%$ is found by evaluating (24) with an equality, the performance degradation is invisible as compared to the mismatch-free case. On the other hand, there is a noticeable performance degradation if we select the mismatch level larger than $0.5 \gamma_{R} \%$. Hence, we conclude that the obtained rule-of-thumb of tolerable mismatch levels is valid for Rayleigh fading channels.

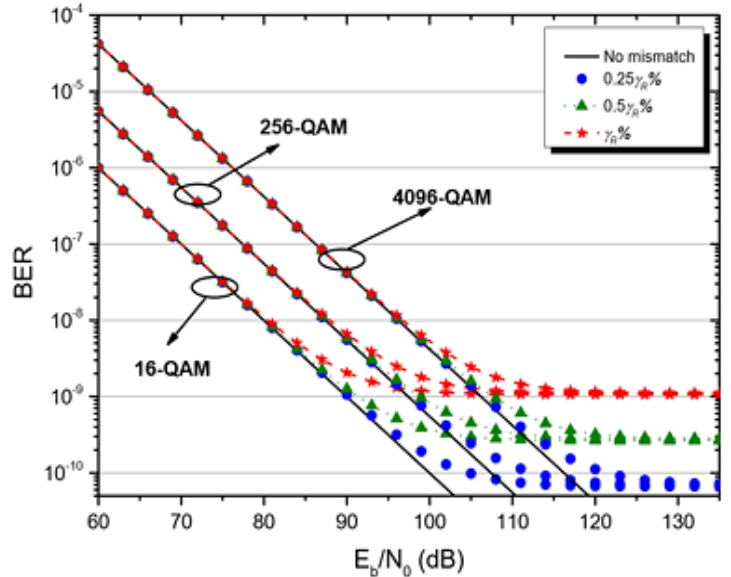

(a)

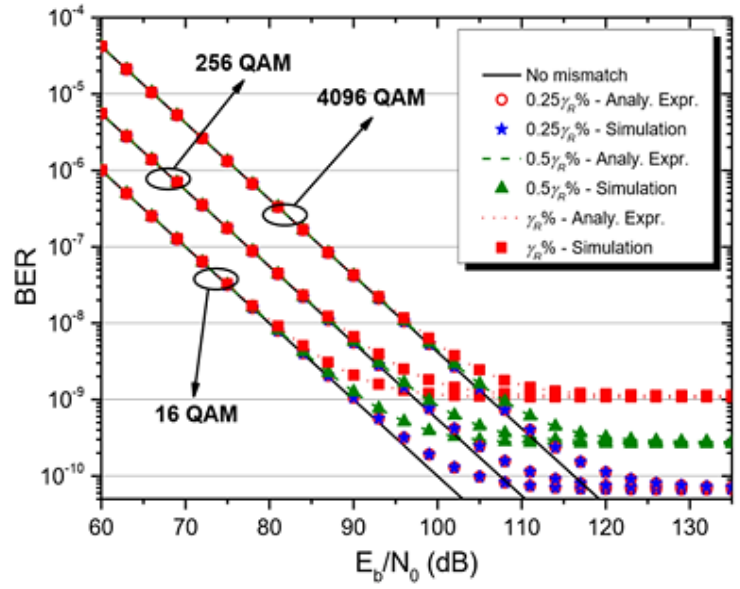

(b)

Figure 3. BER system performance for a Rayleigh channel with $N=2048$ and $L=8$ : (a) fixed timing error values $d t_{l}$ and (b) $10^{10}$ sets of TI-ADC realizations

\section{CONCLUSION}

In this paper, the maximum threshold of TI-ADC timing mismatch level, for which error floors induced by the mismatch is not greater than a considered BER value, has been theoretically identified for OFDM systems in Rayleigh channels. Further, we have revealed that when the mismatch level is selected to be smaller than $25 \%$ of the reliably recommended threshold, the BER performance degradation is not greater than $0.5 \mathrm{~dB}$ as compared to the case without mismatch. This tolerable timing mismatch level can serve as a significant instruction for circuits-and-systems designing engineers to calibrate the TI-ADC timing mismatch through digital signal processing or hardware circuits.

\section{REFERENCES}

[1] L. Zhang, A. Ijaz, P. Xiao, M. M. Molu, and R. Tafazolli, "Filtered OFDM systems, algorithms, and performance analysis for 5G and beyond," IEEE Transactions on Communications, vol. 66, no. 3, pp. 1205-1218, Mar. 2018, doi: 10.1109/TCOMM.2017.2771242.

[2] G. Kongara, C. He, L. Yang, and J. Armstrong, "A comparison of CP-OFDM, PCC-OFDM and UFMC for 5G uplink communications," IEEE Access, vol. 7, pp. 157574-157594, 2019, doi: 10.1109/ACCESS.2019.2949792.

[3] A. J. Seeds, H. Shams, M. J. Fice, and C. C. Renaud, "TeraHertz photonics for wireless communications," Journal of Lightwave Technology, vol. 33, no. 3, pp. 579-587, Feb. 2015, doi: 10.1109/JLT.2014.2355137.

[4] S. Jia et al., "2×300 Gbit/s line rate PS-64QAM-OFDM THz photonic-wireless transmission," Journal of Lightwave Technology, vol. 38, no. 17, pp. 4715-4721, Sep. 2020, doi: 10.1109/JLT.2020.2995702.

[5] W. C. Black and D. A. Hodges, "Time interleaved converter arrays," IEEE Journal of Solid-State Circuits, vol. 15, no. 6, pp. 1022-1029, Dec. 1980, doi: 10.1109/JSSC.1980.1051512.

[6] R. Khoini-Poorfard, L. B. Lim, and D. A. Johns, "Time-interleaved oversampling A/D converters: theory and practice," IEEE Transactions on Circuits and Systems II: Analog and Digital Signal Processing, vol. 44, no. 8, pp. 634-645, 1997, doi: $10.1109 / 82.618037$.

[7] D. Fu, K. C. Dyer, S. H. Lewis, and P. J. Hurst, "A digital background calibration technique for time-interleaved analog-to-digital converters," IEEE Journal of Solid-State Circuits, vol. 33, no. 12, pp. 1904-1911, 1998, doi: 10.1109/4.735530.

[8] J. Li, S. Wu, Y. Liu, N. Ning, and Q. Yu, "A digital timing mismatch calibration technique in time-interleaved ADCs," IEEE Transactions on Circuits and Systems II: Express Briefs, vol. 61, no. 7, pp. 486-490, Jul. 2014, doi: 10.1109/TCSII.2014.2327333.

[9] B. T. Reyes, R. M. Sanchez, A. L. Pola, and M. R. Hueda, "Design and experimental evaluation of a time-interleaved ADC calibration algorithm for application in high-speed communication systems," IEEE Transactions on Circuits and Systems I: Regular Papers, vol. 64, no. 5, pp. 1019-1030, May 2017, doi: 10.1109/TCSI.2016.2636209. 
[10] A. Petraglia and S. K. Mitra, "Analysis of mismatch effects among A/D converters in a time-interleaved waveform digitizer," IEEE Transactions on Instrumentation and Measurement, vol. 40, no. 5, pp. 831-835, 1991, doi: 10.1109/19.106306.

[11] C. Vogel, "The impact of combined channel mismatch effects in time-interleaved ADCs," IEEE Transactions on Instrumentation and Measurement, vol. 54, no. 1, pp. 415-427, Feb. 2005, doi: 10.1109/TIM.2004.834046.

[12] C. A. Schmidt, J. E. Cousseau, J. L. Figueroa, B. T. Reyes, and M. R. Hueda, "Efficient estimation and correction of mismatch errors in time-interleaved ADCs," IEEE Transactions on Instrumentation and Measurement, vol. 65, no. 2, pp. 243-254, Feb. 2016, doi: 10.1109/TIM.2015.2490378.

[13] S. Xu, Y. C. Lim, and J. W. Lee, "Recursive filters for time-interleaved ADC mismatch compensation," IEEE Transactions on Circuits and Systems I: Regular Papers, vol. 63, no. 6, pp. 848-858, Jun. 2016, doi: 10.1109/TCSI.2016.2538061.

[14] S. Chen, L. Wang, H. Zhang, R. Murugesu, D. Dunwell, and A. C. Carusone, "All-digital calibration of timing mismatch error in time-interleaved analog-to-digital converters," IEEE Transactions on Very Large Scale Integration (VLSI) Systems, vol. 25, no. 9, pp. 2552-2560, Sep. 2017, doi: 10.1109/TVLSI.2017.2703141.

[15] P. Monsurro, F. Rosato, and A. Trifiletti, "New models for the calibration of four-channel time-interleaved ADCs using filter banks," IEEE Transactions on Circuits and Systems II: Express Briefs, vol. 65, no. 2, pp. 141-145, Feb. 2018, doi: 10.1109/TCSII.2017.2662084.

[16] M. Guo, J. Mao, S.-W. Sin, H. Wei, and R. P. Martins, “A 1.6-GS/s 12.2-mW seven-/eight-way split time-interleaved SAR ADC achieving 54.2-dB SNDR with digital background timing mismatch calibration," IEEE Journal of Solid-State Circuits, vol. 55, no. 3, pp. 693-705, Mar. 2020, doi: 10.1109/JSSC.2019.2945298.

[17] H.-W. Kang, H.-K. Hong, S. Park, K.-J. Kim, K.-H. Ahn, and S.-T. Ryu, "A sign-equality-based background timing-mismatch calibration algorithm for time-interleaved ADCs," IEEE Transactions on Circuits and Systems II: Express Briefs, vol. 63, no. 6 , pp. 518-522, Jun. 2016, doi: 10.1109/TCSII.2016.2530819.

[18] Y. Oh and B. Murmann, "System embedded ADC calibration for OFDM receivers," IEEE Transactions on Circuits and Systems I: Regular Papers, vol. 53, no. 8, pp. 1693-1703, Aug. 2006, doi: 10.1109/TCSI.2006.879063.

[19] S. Ponnuru, M. Seo, U. Madhow, and M. Rodwell, "Joint mismatch and channel compensation for high-speed OFDM receivers with time-interleaved ADCs," IEEE Transactions on Communications, vol. 58, no. 8, pp. 2391-2401, Aug. 2010, doi: 10.1109/TCOMM.2010.08.090693.

[20] K. M. Tsui and S. C. Chan, "Iterative correction of frequency response mismatches in time-interleaved ADCs: A novel framework and case study in OFDM systems," in The 2010 International Conference on Green Circuits and Systems, Jun. 2010, pp. 253258, doi: 10.1109/ICGCS.2010.5543057.

[21] V.-T.-D. Huynh, N. Noels, and H. Steendam, "Closed-form BER expression for OFDM with the effect of TI-ADC's timing mismatch," in 2018 25th International Conference on Telecommunications (ICT), Jun. 2018, pp. 132-137, doi: 10.1109/ICT.2018.8464910.

[22] V. Huynh, N. Noels, P. Rombouts, J. Armstrong, and H. Steendam, "Effect of time-interleaved analog-to-digital converter mismatches on OFDM performance," in OFDM 2014; 18th International OFDM Workshop 2014 (InOWo'14), 2014, pp. 1-8.

[23] V.-T.-D. Huynh, N. Noels, and H. Steendam, "BER evaluation of OFDM systems with joint effect of TI-ADC circuit's gain mismatch and channel estimation error," IEEE Transactions on Communications, vol. 67, no. 5, pp. 3612-3623, May 2019, doi: 10.1109/TCOMM.2019.2899634.

[24] M. Gardner, Knotted doughnuts and other mathematical entertainments. W.H. Freeman, 1986.

[25] I. Guvenc, S. Gezici, Z. Sahinoglu, and U. C. Kozat, Eds., Reliable communications for short-range wireless systems. Cambridge: Cambridge University Press, 2011

[26] K. Cho and D. Yoon, "On the general BER expression of one- and two-dimensional amplitude modulations," IEEE Transactions on Communications, vol. 50, no. 7, pp. 1074-1080, Jul. 2002, doi: 10.1109/TCOMM.2002.800818.

[27] A. Jeffrey and D. Zwillinger, "Preface to the sixth edition," in Table of Integrals, Series, and Products, Elsevier, 2000. 